\title{
Spontaneous symmetry breaking in amnestically induced persistence
}

\author{
Marco Antonio Alves da Silva, ${ }^{\dagger}$ G. M. Viswanathan ${ }^{\ddagger}$, A. S. Ferreira ${ }^{\ddagger}$ and J. C. Cressoni ${ }^{\ddagger}$ \\ ${ }^{\dagger}$ Departamento de Física e Química, FCFRP, \\ Universidade de São Paulo, 14040-903 Ribeirão Preto, SP, Brazil \\ ${ }^{\ddagger}$ Instituto de Física, Universidade Federal de Alagoas, Maceió-AL, 57079-970, Brazil
}

(Date: August 8, 2021)

\begin{abstract}
We investigate a recently proposed non-Markovian random walk model characterized by loss of memories of the recent past and amnestically induced persistence. We report numerical and analytical results showing the complete phase diagram, consisting of 4 phases, for this system: (i) classical nonpersistence, (ii) classical persistence (iii) log-periodic nonpersistence and (iv) logperiodic persistence driven by negative feedback. The first two phases possess continuous scale invariance symmetry, however log-periodicity breaks this symmetry. Instead, log-periodic motion satisfies discrete scale invariance symmetry, with complex rather than real fractal dimensions. We find for log-periodic persistence evidence not only of statistical but also of geometric self-similarity.
\end{abstract}

Nonpersistent random walkers with negative feedback tend not to repeat past behavior [1, but what happens when they forget their recent past [2]? Remarkably, they become persistent for sufficiently large memory losses. This recently reported phenomenon of amnestically induced persistence [2, 3] allows log-periodic [4] superdiffusion [5, 6, 7] driven by negative feedback. Its practical importance stems from the conceptual advance of quantitatively relating, on a causal level, two otherwise apparently unconnected phenomena: repetitive or persistent behavior on the one hand, and recent memory loss on the other, e.g., in Alzheimer's disease 2]. Precisely how does persistence depend on recent memory loss? Here, we answer this question and report numerical and analytical results showing the complete phase diagram for the problem, comprising 4 phases: (i) classical nonpersistence (ii) classical persistence, (iii) log-periodic nonpersistence and (iv) log-periodic persistence driven by negative feedback. The former two phases possess continuous scale invariance symmetry, which breaks down in the other two.

Random walkers without memory have a mean square displacement $\left\langle x^{2}\right\rangle$ that scales with time $t$ according to $\left\langle x^{2}\right\rangle \sim t^{2 H}$, with Hurst exponent $H=1 / 2$ as demanded by the Central Limit Theorem, assuming finite moments. Hurst exponents $H>1 / 2$ indicate persistence and can arise due to long-range memory. Most random walks with and without memory display continuous scale invariance symmetry, i.e., continuous scale transformations by a "zoom" factor $\lambda$ leave the Hurst exponent unchanged: $t^{2 H} \rightarrow \lambda^{2 H} t^{2 H}$ as $t \rightarrow \lambda t$.

Schütz and Trimper [1] pioneered a novel approach for studying walks with long-range memory [6, 7, 8, ,9], which we have adapted [2] for studying memory loss. Consider a random walker that starts at the origin at time $t_{0}=0$, with memory of the initial $f t$ time steps of its complete history $(0 \leq f \leq 1)$. At each time step the random walker moves either one step to the right or left. Let $v_{t}= \pm 1$ represent the "velocity" at time $t$, such that the position follows

$$
x_{t+1}=x_{t}+v_{t+1} .
$$

At time $t$, we randomly choose a previous time $1 \leq t^{\prime}<$ $f t$ with equal a priori probabilities. The walker then chooses the current step direction $v_{t}$ based on the value of $v_{t^{\prime}}$, using the following rule. With probability $p$ the walker repeats the action taken at time $t^{\prime}$, and with probability $1-p$ the walker takes a step in the opposite direction $-v_{t^{\prime}}$. Values $p>1 / 2(p<1 / 2)$ generate positive (negative) feedback. For $p$ sufficiently larger than $p=1 / 2$, the behavior becomes persistent (i.e., $H>1 / 2$ ). But the finding of persistence for $p<1 / 2$ and small $f$ overturned commonly held beliefs concerning repetitive behavior and memory loss [2]. Very recently, Kenkre [3] has found an exact solution to this problem for the behavior of the first moment, for all $f$, and generalized it in important ways, with excellent agreement with the numerical results over 6 orders of magnitude in time. Here, we investigate how persistence depends quantitatively on memory loss and how to characterize the important underlying symmetry properties.

Fig. 1(a) shows values of $H(f, p)$, estimated via simulations, as a function of the feedback parameter $p$ and the memory fraction $f$. We choose an order parameter $2 H-1$ that has positive values only in the persistent regime. Misleadingly, only two phases may at first seem apparent, namely, persistent and nonpersistent. However, the persistent regime comprises two different phases with distinct symmetry properties. For $p>1 / 2$ we find classical persistence satisfying continuous scale invariance symmetry. In contrast, we find discrete scale invariance symmetry [4] for $p<1 / 2$ : scale invariance holds only for discrete values of the "zoom" or magnification $\lambda_{k}=\lambda^{k}$ $(k=1,2,3 \ldots)$. The nonpersistent regime, similarly, allows for monotonically growing as well as log-periodic 
solutions. The simulation results agree well with our analytical results discussed further below (see Fig. 1(b)). This spontaneous symmetry breaking indicates a distinct phase (and eliminates the possibility of an infinite-order phase transition) 10]. Mathematically, discrete scale invariance symmetry [4] involves complex fractal [11, 12] dimensions: for $z=a+b i$, the real part of $t^{z}$ equals $t^{a} \cos [b \log t]$, indicating log-periodicity [4]. Fig. 1(c) parametrically plots two independent persistent walks for $p<1 / 2(p=0.1$ and $f=0.1)$. The walks appear not only statistically but also geometrically self-similar. Indeed, we find a pattern reminiscent of a logarithmic spiral. We estimate a value of the critical exponent $\beta=1$ from the double log plot (Fig. 1(d)) of the order parameter versus $\left|p-p_{c}\right|$.

We next approach the problem analytically. We choose $v_{t^{\prime}}= \pm 1$ and use the previously discussed recurrence relation, Eq. 1. Let $n_{f}(t)$ e $n_{b}(t)$ denote the number of steps taken in the forward and backward directions respectively, at time $t$ (inclusive). The total number of steps is thus $n_{f}(t)+n_{b}(t)=t$. For full memory, the probability to take a step in the forward direction at time $t+1$, for $t \geq 1$, is $P_{\mathrm{eff}}^{+}(t)=\frac{n_{f}(t)}{t} p+\frac{n_{b}(t)}{t}(1-p)$. Similarly,

$P_{\text {eff }}^{-}(t)=\frac{n_{b}(t)}{t} p+\frac{n_{f}(t)}{t}(1-p)$. So, the effective value expected at time $t+1$ is $v_{t+1}^{e}=P_{\text {eff }}^{+}(t)-P_{\text {eff }}^{-}(t)$. Since $x_{t}=n_{f}(t)-n_{b}(t)+x_{0}$, we obtain $v_{t+1}^{e}=\alpha \frac{x_{t}-x_{0}}{t}$, where $\alpha=2 p-1$. We can interpret this result as a series of experiments or walks at time $t$ having the same number of steps forwards and backwards, giving the value $v_{t+1}^{e}$.

Now we introduce memory loss. Let the memory range be $L=L(t)=\operatorname{int}(f t)$, where $\operatorname{int}(x)$ denotes the integer part of $x$, for $0<f \leq 1$, starting at $t=0$. In analogy to the results above, we obtain $v_{t+1}^{e}=\alpha \frac{x_{L}-x_{0}}{L}$.

Now we study the $n$th moments of $x_{t}^{n}$. Taking Eq. 1 to power $n$ we get $x_{t+1}^{n}=\left(x_{t}+v_{t+1}\right)^{n}=\sum_{i=0}^{n}\left(\begin{array}{c}n \\ i\end{array}\right) v_{t+1}^{i} x_{t}^{n-i}$. For all even exponents $i$ we know $v_{t+1}^{i}=1$ and for odd exponents $v_{t+1}^{i}=v_{t+1}$. Using the expression for $v_{t+1}^{e}$, with $x_{0}=0$, we obtain

$$
\begin{aligned}
& \left\langle x_{t+1}^{n}\right\rangle=\Delta+\left\langle x_{t}^{n}\right\rangle+\frac{n \alpha}{L}\left\langle x_{L} x_{t}^{n-1}\right\rangle+ \\
& \sum_{l=1}^{s(n)}\left[\left(\begin{array}{c}
n \\
2 l
\end{array}\right)\left\langle x_{t}^{n-2 l}\right\rangle+\frac{\alpha}{L}\left(\begin{array}{c}
n \\
2 l+1
\end{array}\right)\left\langle x_{L} x_{t}^{n-2 l-1}\right\rangle\right]
\end{aligned}
$$

where $\Delta=\frac{1+(-1)^{n}}{2}$ and $s(n)=\frac{n-\Delta-1}{2}$. We have $\Delta=1$ for even $n$ and $\Delta=0$ for odd $n$. If $s(n)<1$, then the sum vanishes.

In the asymptotic limit, we arrive at the following differential equation for the moments, starting from Eq. 2.

$$
\frac{d}{d t}\left\langle x_{t}^{n}\right\rangle=\Delta+\frac{n \alpha}{L}\left\langle x_{L} x_{t}^{n-1}\right\rangle+
$$
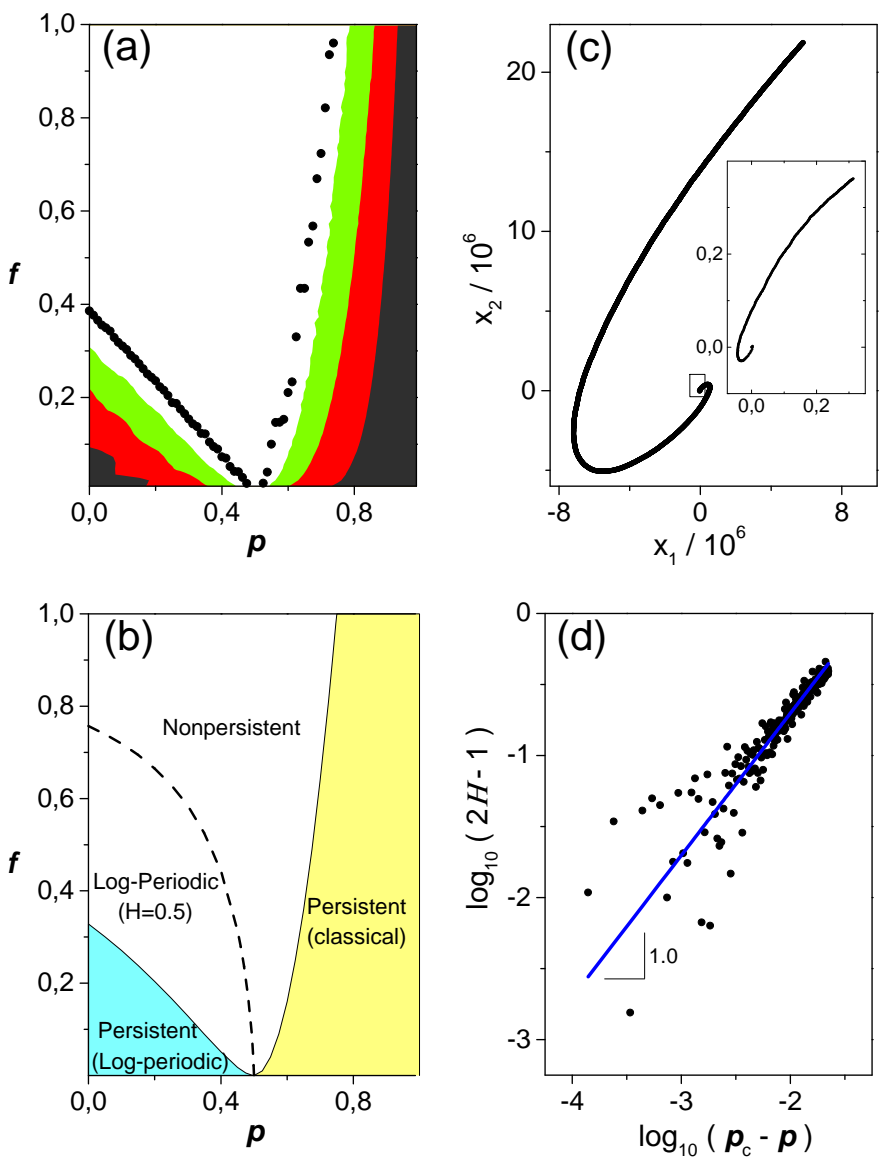

FIG. 1: (a) Hurst exponent $H(p, f)$ estimated from simulations of non-Markovian walks that remember only a fraction $f$ of their distant past, with feedback parameter $p$. Color scheme: $H<0.6$ (white), $0.6<H<0.7$ (green), $0.7<H<0.9$ (red), $H>0.9$ (black). Full circles show the edge where $H=1 / 2$. Persistence $(H>1 / 2)$ arises for positive $(p>1 / 2)$ as well as negative $(p<1 / 2)$ feedback. (b) complete phase diagram showing the 4 phases, plotted according to the exact solutions given by Eqs. 11] 13. The dashed line $f_{0}(p)$ delineates the threshold for log-periodicity and cleaves the nonpersistent regime into two (Eq. 8). (c) parametric plot of positions $x_{1}(t)$ and $x_{2}(t)$ for two realizations of log-periodic walks. Inset shows zoom of the center (boxed area) of the logarithmic-like spiral. Note the geometric self-similarity. (d) double log plot of $2 H-1$ versus $p_{c}-p$, showing critical behavior with critical exponent $\beta=1$.

$$
\sum_{l=1}^{s(n)}\left[\left(\begin{array}{c}
n \\
2 l
\end{array}\right)\left\langle x_{t}^{n-2 l}\right\rangle+\frac{\alpha}{L}\left(\begin{array}{c}
n \\
2 l+1
\end{array}\right)\left\langle x_{L} x_{t}^{n-2 l-1}\right\rangle\right] .
$$

For the first moment $(n=1)$, we obtain an equation identical to the one obtained by Kenkre [3] :

$$
\frac{d}{d t}\left\langle x_{t}\right\rangle=\frac{\alpha}{f t}\left\langle x_{f t}\right\rangle
$$

Considering an expansion of the form 


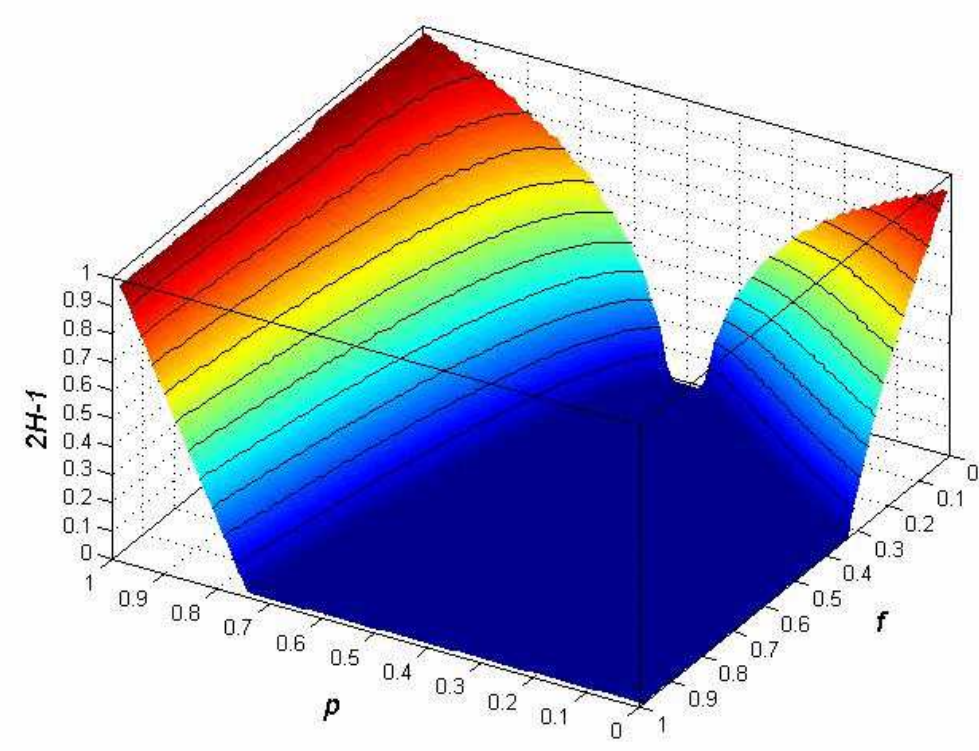

FIG. 2: The order parameter $2 H-1$ versus $p$ and $f$, estimated using Eqs. 10 and 12

$\left\langle x_{t}\right\rangle \sim \sum_{i} A_{i} t^{\delta_{i}} \sin \left(B_{i} \ln (t)+C_{i}\right)$, we obtain a system of transcendental equations for $B$ and $\delta$ :

$$
\begin{aligned}
\delta & =\alpha f^{\delta-1} \cos (B \ln f) \\
B & =\alpha f^{\delta-1} \sin (B \ln f) .
\end{aligned}
$$

For $\alpha \geq 0$, we have $B=0$. The system given by Eqs. [5] and 6] reduces to Eq. 7 below, ruling out a logperiodic solution. For $\delta>1 / 2$, we obtain superdiffusion (see below).

For $\alpha<0$, there exists a threshold defined by a continuous set of values $(p, f)$, with oscillating solutions. Consider first the case $B=0$, without oscillations. Then, Eq. 5 becomes

$$
\delta=\alpha f^{\delta-1}
$$

which only has solutions for $f>f_{0}(p)$. Numerically solving this equation, we find the threshold $f_{0}(0)=0.7569$ for $p=0$, in perfect agreement with the expression

$$
(1-2 p) \ln \left(1 / f_{0}\right)=f_{0} / e,
$$

obtained by Kenkre (personal communication, 13 July 2007) for the onset of log-periodicity [3], shown as a dashed line in Fig 1(b). Indeed, we have found an alternative proof of Eq. 8 starting from Eq. 7 using the Lambert $\mathrm{W}$ function. For $\delta>1 / 2$, both superdiffusion and log-periodicity appear (see below).

We next study the second moment. If $n=2$ then $\Delta=1$ and $s(n)=0$. Thus, Eq. 3 leads to

$$
\frac{d}{d t}\left\langle x_{t}^{2}\right\rangle=1+\frac{2 \alpha}{f t}\left\langle x_{f t} x_{t}\right\rangle
$$

Using the fact that $|\langle x\rangle| \leq\left(\left\langle x^{2}\right\rangle\right)^{1 / 2}$, we can prove that there exists a function $A(t)$ such that $\left\langle x_{f t} x_{t}\right\rangle=$ $A(t)\left(\left\langle x_{f t}^{2}\right\rangle\left\langle x_{t}^{2}\right\rangle\right)^{1 / 2}$, with $-1 \leq A(t) \leq 1$.

For $\alpha \geq 0(p \geq 1 / 2)$, no oscillations appear and $A(t \rightarrow \infty)=1$. We can thus show that the following transcendental relationship holds for the Hurst exponent, asymptotically:

$$
H=\alpha f^{H-1} .
$$

This result is identical to Eq. [7 with $\delta=H$. Consequently, the curve

$$
f_{c}=16\left(p_{c}-\frac{1}{2}\right)^{2}
$$

separates the diffusive and anomalous regions for $p \geq 1 / 2$ in the $(p, f)$ plane (Fig. 1(b)). The case $f=1$ leads to $p_{c}=3 / 4$, in agreement with ref. [1].

For $\alpha<0$, we try the expansion $\left\langle x_{t}^{2}\right\rangle \sim \sum_{i} a_{i} t^{2 H_{i}} \sin ^{2}\left(b_{i} \ln t+c_{i}\right)$. We assume that the dominant terms of $\left\langle x_{t}\right\rangle$ and $\left\langle x_{t}^{2}\right\rangle$ have the same "period" and phase difference, so that $b=B$ and $c=C$. In the log-periodic region (i.e. $b \neq 0$ ), we also assume that $H \geq \delta$, so that the solution follows from Eqs. 5 e 6 . Indeed we conjecture that for walks lacking subdiffusion, $\delta \geq 1 / 2$ implies $H=\delta$ always. Subject to natural restrictions, the Hurst exponent must thus satisfy

$$
H=\frac{\sqrt{\alpha^{2} f^{2 H-2}-H^{2}}}{\tan \left[\ln (f) \sqrt{\alpha^{2} f^{2 H-2}-H^{2}}\right]}
$$

for $\delta \geq 1 / 2$ and $H=1 / 2$ otherwise. The separation line of the diffusive and anomalous phases corresponds to $H=\delta=1 / 2$, so the critical line (Fig. 1(b)) satisfies

$$
2 \sqrt{\frac{\alpha_{c}^{2}}{f_{c}}-\frac{1}{4}}=\tan \left[\ln \left(f_{c}\right) \sqrt{\frac{\alpha_{c}^{2}}{f_{c}}-\frac{1}{4}}\right]
$$

We obtain the critical value of $f_{c}(0)=0.3284$ for the onset of $\log$-periodic superdiffusion, which occurs at $p=0$. Note that $p_{c}=1 / 2, f_{c}=0$ represents a multicritical point. Fig. 11(b) shows the complete phase diagram, consisting of 4 phases. Fig. 2 shows a better view of how the order parameter $2 H-1$ depends on $p$ and $f$.

Substituting our choice of $\left\langle x_{t}^{2}\right\rangle$ into Eq. 9, with the definition of $A(t)$, we obtain

$$
A(t)=\frac{H \sin (b \ln t+c)^{2}+b \sin (b \ln t+c) \cos (b \ln t+c)}{\alpha f^{H-1}|\sin (b \ln t+c)||\sin (b \ln f t+c)|} .
$$

Semi-empirically, we find that $A(t) \approx \pm 1$. Specifically, we propose

$$
A(t)=\frac{\sin (b \ln t+c) \cos (b \ln f t+c)}{|\sin (b \ln t+c) \sin (b \ln f t+c)|} .
$$


Note that $A(t)=1$ for $f=1$. Applying the same reasoning for the case $H=1 / 2$, we obtain

$$
\begin{aligned}
A(t)= & f^{1 / 2}[2 \alpha a|\sin (b \ln t+c)||\sin (b \ln f t+c)|]^{-1} \\
& \times\left[a \sin (b \ln t+c)^{2}\right. \\
& +2 a b \sin (b \ln t+c) \cos (b \ln t+c)-1] .
\end{aligned}
$$

Thus, exactly on the critical line, we get a marginally superdiffusive solution: $\left\langle x^{2}(t)\right\rangle=a t \ln t \sin ^{2}(b \ln t+c)$. simulations suggest that higher order terms in the expansion become important near the critical line.

We next focus on finding a suitable Fokker-Planck Equation (FPE). Let $Y$ denote the position of a walker. Consider the conditional probability at position $Y$ at time $t+1$, given position $x_{0}$ at time $t=0$ :

$$
\begin{aligned}
P\left(Y, t+1 \mid x_{0}, 0\right) & =P\left(Y+1, t \mid x_{0}, 0\right) P_{\mathrm{eff}}^{b}(t, Y+1) \\
& +P\left(Y-1, t \mid x_{0}, 0\right) P_{\mathrm{eff}}^{f}(t, Y-1) .
\end{aligned}
$$

Using the definitions of $n_{f}(t)$ and $n_{b}(t)$, and the probabilities to go forwards or backwards, we obtain

$$
P_{\mathrm{eff}}^{ \pm}(t, Y)=\frac{1}{2}\left[1 \pm \frac{\alpha\left(Y-x_{0}\right)}{t}\right] .
$$

Substituting Eq. 18 in Eq. 17, we get

$$
\begin{aligned}
& P\left(Y, t+1 \mid x_{0}, 0\right) \\
& =\frac{1}{2}\left[1-\frac{\alpha\left(Y-x_{0}+1\right)}{t}\right] P\left(Y+1, t \mid x_{0}, 0\right) \\
& +\frac{1}{2}\left[1+\frac{\alpha\left(Y-x_{0}-1\right)}{t}\right] P\left(Y-1, t \mid x_{0}, 0\right) .
\end{aligned}
$$

From this last equation, in the asymptotic limit for $t$ and $Y$, we get a FPE for $x=Y$ which is identical to the one in ref. 1], as expected. Similarly, we can show for memory loss that

$$
\frac{\partial P(x, t)}{\partial t}=\frac{1}{2} \frac{\partial^{2}}{\partial x^{2}} P(x, t)-\frac{\alpha}{f t} \frac{\partial}{\partial x}\left[x_{f t} P(x, t)\right],
$$

where $x_{f t}$ denotes the position at time $f t$ that leads to position $x$ in the future, at time $t$.

In summary, we have uncovered the essential features of the phase diagram for this problem, based on numerical as well as analytical results. We expect the phase diagram and other findings reported here to contribute towards a better quantitative description of persistence in diverse economic 13], sociological 14], ecological [15], biological [5, 15] and physiological [5] complex systems where recent memory loss may play a role [2]. Specifically, prime candidates for further study include systems with long-range memory that show evidence of logperiodicity and discrete scale invariance symmetry [4]. The most important result, for systems with negative feedback, concerns the existence of a critical threshold of memory loss for the onset of superdiffusion. Finally, we hope that the insights provided by this study dispel lingering doubts that negative feedback coupled with recent memory loss can in fact cause persistence.

\section{Acknowledgements}

We thank V. M. Kenkre and Marcelo L. Lyra for discussions and CNPq and FAPESP for financial assistance.

[1] G. M. Schütz and S. Trimper, Phys. Rev. E 70, 045101 (2004).

[2] J. C. Cressoni, M. A. A. da Silva, and G. M. Viswanathan, Phys. Rev. Lett. 98, 070603 (2007).

[3] V. M. Kenkre, "Analytic Formulation, Exact Solutions, and Generalizations of the Elephant and the Alzheimer Random Walks," arXiv:0708.0034 v2 [condmat.stat-mech] 6 Aug 2007.

[4] D. Sornette, Proc. Nat. Acad. Sci. 99, 2522 (2002).

[5] Lévy flights and related topics in physics, edited by $\mathrm{M}$. F. Shlesinger, G. M. Zaslavsky and U. Frisch (Springer, Berlin, 1995).

[6] R. Metzler and J. Klafter, Phys. Rep. 339, 1 (2000).

[7] R. Metzler and J. Klafter, J. Phys. A 37, R161 (2004).

[8] V. M. Kenkre, in Statistical Mechanics and Statistical Methods in Theory and Application, edited by U. Landman (Plenum, New York, 1977).

[9] V. M. Kenkre, E. W. Montroll and M. F. Shlesinger, J. Stat. Phys. 9, 45 (1973).

[10] H. E. Stanley, Introduction to Phase Transitions and Critical Phenomena (Oxford University Press, Oxford and New York, 1971).

[11] B. B. Mandelbrot, The Fractal Geometry of Nature (Freeman, San Fransisco, 1982).

[12] Bunde A., and S. Havlin, Fractals and Disordered Systems (Springer, Berlin, 1991).

[13] R. N. Mantegna and H. E. Stanley, An Introduction to Econophysics (Cambridge Univ. Press, Cambridge 2000).

[14] B. J. West, Mathematical Models As a Tool for the Social Sciences (Taylor and Francis, London, 1980).

[15] P. Turchin, Quantitative Analysis of Movements: Measuring and Modeling Population Redistribution in Animals and Plants (Sinauer Associates, Sunderland, 1998). 